\title{
Prediction of Suicide and Nonfatal Self-harm After Bariatric
}

Surgery: A Risk Score Based on Sociodemographic Factors, Lifestyle Behavior, and Mental Health : A Nonrandomized Controlled Trial

\section{Konttinen, Hanna}

2021-08

Konttinen , H , Sjöholm , K , Jacobson , P , Svensson, P-A , Carlsson , L \& Peltonen , M 2021 , ' Prediction of Suicide and Nonfatal Self-harm After Bariatric Surgery: A Risk Score Based on Sociodemographic Factors, Lifestyle Behavior, and Mental Health : A Nonrandomized Controlled Trial ' , Annals of Surgery , vol. 274 , no. 2 , pp. 339-345 . https://doi.org/10.1097/SLA.00

cc_by_nc

acceptedVersion

Downloaded from Helda, University of Helsinki institutional repository.

This is an electronic reprint of the original article.

This reprint may differ from the original in pagination and typographic detail.

Please cite the original version. 
Prediction of Suicide and Non-Fatal Self-Harm After Bariatric Surgery - A Risk Score Based on Sociodemographic Factors, Lifestyle Behavior and Mental Health A Non-Randomized Controlled Trial

Hanna Konttinen, $\mathrm{PhD}^{1,2}$; Kajsa Sjöholm, $\mathrm{PhD}^{3}$; Peter Jacobson, $\mathrm{MD}, \mathrm{PhD}^{3}$; Per-Arne Svensson, $\mathrm{PhD}^{3,4}$; Lena M. S. Carlsson, $\mathrm{MD}, \mathrm{PhD}^{3}$; Markku Peltonen, $\mathrm{PhD}^{5}$

${ }^{1}$ Faculty of Social Sciences, University of Helsinki, Helsinki, Finland

${ }^{2}$ Department of Food and Nutrition, University of Helsinki, Helsinki, Finland

${ }^{3}$ Institute of Medicine, the Sahlgrenska Academy at the University of Gothenburg,

Gothenburg, Sweden

${ }^{4}$ The Institute of Health and Care Sciences, the Sahlgrenska Academy at the University of Gothenburg, Gothenburg, Sweden

${ }^{5}$ Public Health Promotion Unit, National Institute for Health and Welfare, Helsinki, Finland

Corresponding author: Dr Hanna Konttinen, Faculty of Social Sciences, University of Helsinki, Unioninkatu 35 (P.O. Box 18), 00014 University of Helsinki, Helsinki, Finland. Email: hanna.konttinen@helsinki.fi, Tel.: +358 294124892.

Reprints: Dr Hanna Konttinen, Faculty of Social Sciences, University of Helsinki, Unioninkatu 35 (P.O. Box 18), 00014 University of Helsinki, Helsinki, Finland. E-mail: hanna.konttinen@helsinki.fi, Tel.: +358 294124892. 


\section{Conflicts of Interest and Source of Funding}

Dr Carlsson has received consulting and lecture fees from Johnson \& Johnson and AstraZeneca. All other authors report no conflicts of interest.

This work was supported by the Academy of Finland (grants 314135 and 309157 to Dr

Konttinen), the National Institute of Diabetes and Digestive and Kidney Diseases of the US National Institutes of Health (NIH; award number R01DK105948), The Swedish Research Council (Grant 2017-01707), and grants from the Swedish state under the agreement between the Swedish government and the country councils, the ALF-agreement (ALFGBG-717881). The study funders had no role in study design; in the collection, analysis, and interpretation of data; in the writing of the report; and in the decision to submit the paper for publication.

Running head: Predicting Self-Harm After Bariatric Surgery 


\section{STRUCTURED ABSTRACT}

Objective: To identify pre-operative sociodemographic and health-related factors that predict higher risk of non-fatal self-harm and suicide after bariatric surgery.

Summary of Background Data: Evidence is emerging that bariatric surgery is related to an increased risk of suicide and self-harm, but knowledge on whether certain pre-operative characteristics further enhance the excess risk is scarce.

Methods: The non-randomized, prospective, controlled Swedish Obese Subjects study was linked to two Nationwide Swedish registers. The bariatric surgery group ( $\mathrm{N}=2007$, perprotocol) underwent gastric bypass, banding or vertical banded gastroplasty, and matched controls (N=2040) received usual care. Participants were recruited from 1987 to 2001, and information on the outcome (a death by suicide or non-fatal self-harm event) was retrieved until the end of 2016. Sub-hazard ratios (subHR) were calculated using competing risk regression analysis.

Results: The risk for self-harm/suicide was almost twice as high in surgical patients compared to control patients both before and after adjusting for various baseline factors (adjusted subHR=1.98, 95\% CI=1.34-2.93). Male sex, previous health-care visits for selfharm or mental disorders, psychiatric drug use, and sleep difficulties predicted higher risk of self-harm/suicide in the multivariate models conducted in the surgery group. Interaction tests further indicated that the excess risk for self-harm/suicide related to bariatric surgery was stronger in men (subHR=3.31, 95\% CI=1.73-6.31) than in women $(\operatorname{subHR}=1.54,95 \%$ $\mathrm{CI}=1.02-2.32)(\mathrm{P}=0.007$ for adjusted interaction). A simple-to-use score was developed to identify those at highest risk of these events in the surgery group.

Conclusions: Our findings suggest that male sex, psychiatric disorder history, and sleep difficulties are important predictors for non-fatal self-harm and suicide in post-bariatric 
patients. High-risk patients who undergo surgery might require regular post-operative psychosocial monitoring to reduce the risk for future self-harm behaviors. 


\section{INTRODUCTION}

Bariatric surgery is currently the most effective treatment for severe obesity and its comorbidities, such as type 2 diabetes and cardiovascular diseases. ${ }^{1-4}$ It also reduces the risk of premature mortality from all-causes and comorbidity-specific causes. ${ }^{5-7}$ Nonetheless, studies have further shown that post-operative outcomes vary between patients with some individuals experiencing adverse effects on their mental health and health-related quality of life (HRQL). Self-harm behaviors, suicidal ideation, suicide attempts, and death by suicide represent the most adverse mental health outcomes and evidence is emerging that bariatric surgery is related to an increased risk of self-harm and suicide..$^{8-11}$ In a meta-analysis of 32 studies (148 643 individuals), the risk of non-fatal self-harm was two times higher after bariatric surgery within the same population and four times higher compared to age-, sex-, and BMI-matched controls. ${ }^{12}$ Prior work has also demonstrated that suicide occurs more frequently after the first or second post-operative year. ${ }^{13}$ Thus, a challenge is to identify patient characteristics that predict these adverse outcomes after bariatric surgery. This would enable targeting post-operative psychosocial monitoring and support for high-risk patients. ${ }^{14}$

However, to date, only a few studies have explored whether certain pre-operative characteristics further pronounce the excess risk of self-harm/suicide related to bariatric surgery. Two prospective register-based cohort studies conducted in Sweden ${ }^{9}$ and Australia ${ }^{10}$ found that a history of hospitalization due to depression or a diagnosis of self-harm before bariatric surgery predicted deliberate self-harm hospitalizations after surgery. Moreover, a study following self-harm emergencies 3 years before and after bariatric surgery observed that nearly all events $(93 \%)$ occurred in patients with a history of mental disorder. ${ }^{8}$ 
We used the Swedish Obese Subjects (SOS) study with extensive baseline information and long-term follow-up after bariatric surgery to extend this scarce literature on potential preoperative risk factors. Recently, we showed that suicides or non-fatal self-harm events were more frequent in the SOS patients undergoing bariatric surgery than in control patients. ${ }^{11}$ The main aims of the present study were 1) to examine which pre-operative sociodemographic and health-related factors predict higher risk of non-fatal self-harm/suicide following bariatric surgery, and 2) to develop a simple-to-use score to identify patients with highest risk for these events in the bariatric surgery group.

\section{METHODS}

Participants and study design

Participants were from the Swedish Obese Subjects (SOS) study, which is a matched, nonrandomized, prospective intervention trial comparing bariatric surgery with usual care for patients with obesity. ${ }^{1,3}$ In the SOS study, 4047 obese patients at 25 surgical departments and 480 primary health-care centers in Sweden were enrolled between September 1, 1987 and January 31, 2001. Patients were recruited from a matching examination that was completed by 6905 patients of which 5335 were eligible for the study inclusion. Age 37-60 years and BMI $\geq 34 \mathrm{~kg} / \mathrm{m}^{2}$ for men and BMI $\geq 38 \mathrm{~kg} / \mathrm{m}^{2}$ for women at recruitment served as the inclusion criteria. The exclusion criteria included earlier surgery for gastric or duodenal ulcer, earlier bariatric surgery, gastric ulcer or myocardial infarction during the past 6 months, ongoing malignancy, active malignancy during the past 5 years, bulimic eating pattern, drug or alcohol abuse, psychiatric or cooperative problems contraindicating bariatric surgery and other contraindicating conditions (e.g. chronic glucocorticoid or anti-inflammatory treatment). 
In the SOS study, 2010 individuals electing the surgery as a co-decision with the physician constituted the surgery group ( $\mathrm{N}=2007$ per protocol as 3 patients were never operated), while 18 matching variables were used to create a contemporaneously matched control group of 2037 individuals ( $N=2040$ per protocol). Even though the surgical patient and the matched control patient always started the study on the day of surgery, the matching was not performed at an individual level. The matching algorithm instead selected controls in a way that the current mean values of the matching variables in the control group became as similar as possible to the current mean values in the surgery group according to the method of sequential treatment assignment. ${ }^{15}$

Surgery type was determined by the surgeon and surgical patients underwent either nonadjustable or adjustable banding $(\mathrm{N}=376)$, vertical banded gastroplasty $(\mathrm{N}=1365)$, or gastric bypass $(\mathrm{N}=266)$. Control patients were offered usual care at their regular primary health-care center. The treatment was not standardized and varied according to the local practices from sophisticated lifestyle intervention and behavior modification to a lack of specific treatment and health monitoring only. All patients attended a health examination and completed various questionnaires prior to intervention.

The study was approved by seven regional ethical review boards in Sweden, and all patients gave written or oral informed consent to participate. The SOS study was registered at clinicaltrials.gov as NCT01479452.

\section{Measures}

Outcome variable 
The primary outcome in the SOS study was all-cause mortality at 10 years of follow-up. ${ }^{6}$ The outcome in the present analysis was a death by suicide or non-fatal self-harm event. Non-fatal self-harm/suicide was not predefined outcome in the original study plan. Information on this outcome was retrieved from the Cause of Death Register and the National Patient Register (NPR) until December 31, 2016. The NPR covered both specialized out-patient and in-patient care since 2001, while it covered only in-patient care before 2001. International Classification of Diseases (ICD) codes were used to identify suicide and non-fatal self-harm events (ICD9 codes E950-959 and E980-989; ICD10 codes X60-84, Y10-34 and Y870), including both confirmed suicides and deaths from undetermined intent.

Pre-treatment predictor variables

Sociodemographic characteristics. Sex and age (years). Education level (1=basic, $2=$ upper secondary, $3=$ university) and marital status $(0=$ non-married or no partner, $1=$ married or partner) were based on self-reported information.

Physical and mental health status. BMI $\left(\mathrm{kg} / \mathrm{m}^{2}\right)$ was calculated based on weight and height measurements (both measured in a standing position without shoes). Diabetes was defined as a fasting blood glucose level of at least $6.1 \mathrm{mmol}$ per litre $(110 \mathrm{mg}$ per decilitre) or selfreported use of a prescribed antidiabetic medication. The presence of work-restricting pain in joints was self-reported. Patients with health-care visits for non-fatal self-harm, substance abuse and mental disorders before participation in the SOS study were identified from the NPR using ICD codes (for details, see Supplemental Table 1). Psychiatric drug use during the last three months was retrieved via self-report ${ }^{16}$ and the responses were classified according to the Anatomical Therapeutic Chemical (ATC) classification system codes (N05: psycholeptics, N06: psychoanaleptics). 
Lifestyle behaviors. Daily smoking and alcohol consumption were measured using self-report questionnaires. The validated SOS dietary questionnaire covered habitual intake of a range of alcoholic beverages during the last three months. ${ }^{17}$ The total average alcohol intake in grams per day was calculated from the responses. Sleep difficulties were defined as awake in bed per night (h) based on two self-report items: the average time spent in bed per night was subtracted from the average sleep time per night. Respondents also filled in the 51-item Three-Factor Eating Questionnaire assessing cognitive restraint of eating, disinhibition, and susceptibility to hunger. ${ }^{18}$ Supplemental Table 2 provides detailed information on these scales and their scoring.

Self-rated HRQL. The following validated self-administered questionnaires were used to assess HRQL and its physical, mental and social dimensions: 1) perceived health status: Current Health scale of the General Health Rating Index $;{ }^{19}$ 2) overall mood: the short version of the Mood Adjective Check List; ${ }^{20}$ 3) depression and anxiety: the Hospital Anxiety and Depression Scale (HADS); ${ }^{21} 4$ ) health-related limitations in social life: the Social Interaction category of the Sickness Impact Profile; ${ }^{22} 5$ ) the impact of obesity on psychosocial functioning: the Obesity-related Problems scale. ${ }^{23}$ For details, see Supplemental Table 2.

\section{Statistical analyses}

Baseline descriptive statistics are presented as mean values with standard deviations or as percentages. Baseline comparisons between the treatment groups used t-tests for continuous variables and Fisher's exact tests for dichotomous variables. The follow-up started on the date of surgery for both the surgical and matched control patient. Participants were followed until 
the first-time diagnosis of suicide or non-fatal self-harm, emigration, death for other causes, or December 31, 2016, at which point the NPR and the Cause of Death Register were complete and the registers were linked.

Cumulative incidence of non-fatal self-harm/suicide events was estimated with competing risk regression models, in which non-suicide deaths were treated as competing events. Persons who emigrated, altered their obesity intervention (bariatric surgery performed in the control group or reversal of the bariatric surgery procedure in the surgery group resulting in normal anatomy), withdrew their consent or were alive at the end of the follow-up were treated as censored observations. Univariate and multivariate models were applied to obtain relative risk estimates as sub-hazard ratios (subHR) with corresponding 95\% confidence intervals (CI). The risk of non-fatal self-harm/suicide in the surgery group compared to the control group was first evaluated in an unadjusted analysis with a single covariate for the treatment group (surgery or control) and in a secondary analysis adjusted for risk factors for self-harm/suicide.

The predictive ability of the risk factors to identify those with non-fatal self-harm/suicide events was evaluated with Harrell's C-statistic, and the calibration of the models was evaluated with graphical means comparing observed and predicted risks. Within the surgery group, a separate prediction model was created and transformed to a simple scoring system to indicate the risk of self-harm/suicide over 10 years. This score was evaluated regarding discrimination and calibration (over 10 and 20 years). Finally, internal validation of the score was conducted using bootstrapping with 5000 replications of individuals sampled with replication, and Harrell's C-statistic was calculated for the risk score model and the score points as derived within the original study cohort. 
All statistical tests were two-sided and $\mathrm{P}$ values of less than 0.05 were considered statistically significant. Analyses were performed with Stata software (StataCorp. 2017. Stata Statistical Software: Release 15. College Station, TX: StataCorp LLC).

\section{RESULTS}

Surgical patients were younger, had higher BMI, and were less likely to have a college or university level education as compared to control patients (Table 1). Daily smoking, history of self-harm, diabetes and work-restricting joint pain were also more frequent in surgical patients than in control patients. Average disinhibition and hunger scores were higher, whereas average cognitive restraint scores were lower in the surgery group than in the control group. Moreover, compared to control patients, surgical patients rated their physical, mental and social HRQL as lower. Baseline characteristics by self-harm/suicide status during followup are shown in Supplemental Table 3.

During a median follow-up of 21.2 years (IQR=17.7-23.8), 98 self-harm/suicide events occurred in the surgery group. In the control group, 50 self-harm/suicide events occurred during a median follow-up of 19.8 years ( $\mathrm{IQR}=16.1-23.2)$. Of these events, 10 and 3 were suicides in the surgery and control group, respectively. In line with our previous results, ${ }^{11}$ the risk of self-harm/suicide was almost twice as high in surgical patients compared to control patients (unadjusted subHR=1.95, 95\% CI: 1.39-2.75) (Figure 1). This association remained after adjustment for all risk factors included in the multivariate model (adjusted subHR=1.98, 95\% CI: 1.34-2.93) (Table 2). In the surgery and control group, impaired self-rated HRQL, history of self-harm, history of mental disorder, psychiatric drug use, sleep difficulties, and 
smoking ( $\mathrm{P}=0.055$ for the control group) at baseline were related to an increased risk of selfharm/suicide in the univariate models (Supplemental Table 4). In addition, male sex, lower BMI, higher alcohol intake, history of substance abuse, and higher susceptibility to hunger predicted these adverse events in surgical patients, whereas joint pain predicted them in control patients. Due to the large number of baseline risk factors analyzed, only variables with $\mathrm{P}<0.05$ in the univariate models (either in the surgery or control group) were entered into multivariate models (Table 2). In the surgery group, 5 risk factors remained as significant predictors in the multivariate model, i.e. history of self-harm, history of mental disorder, psychiatric drug use, sleep difficulties, and male sex were related to a higher risk of non-fatal self-harm/suicide. In the control group, history of mental disorder and sleep difficulties remained as significant predictors. The Harrel's C-statistics of these regression models were 0.80 (95\% CI: $0.75-0.84)$ for the surgery group and 0.79 (95\% CI: $0.72-0.86)$ for the control group.

We tested significance of interaction terms to evaluate whether the baseline risk factors modified the association between bariatric surgery and subsequent self-harm/suicide events. As shown in Figure 1, sex modified this association $(\mathrm{P}=0.0495$ for unadjusted interaction and $\mathrm{P}=0.007$ adjusted for age, study inclusion year, history of self-harm, history of mental disorder, psychiatric drug use, and sleep difficulties): the excess risk of self-harm/suicide related to bariatric surgery was more pronounced in men ( $\operatorname{subHR}=3.31,95 \% \mathrm{CI}: 1.73-6.31$ ) than in women ( $\mathrm{subHR}=1.54,95 \% \mathrm{CI}: 1.02-2.32)$. Other sociodemographic factors, physical or mental health status, lifestyle behaviors, or self-rated HRQL did not have significant modifying effects (Supplemental Figures 1 and 2). 
Finally, in order to develop a risk score, we estimated a separate multivariate regression model for self-harm/suicide events in the surgery group and used the regression coefficients in a score point system (Table 3 ). Total points in the resulting score varied between 0 and 13 with higher values indicating higher risk. A calibration plot (Supplemental Figure 3) comparing the predicted risk and the corresponding observed risk indicated good agreement. Among those with the highest risk, however, the prediction over-estimated the observed risk slightly. In the internal validation with bootstrap samples, the discrimination was reasonably good both for the original model underlying the score (the mean C-statistic 0.78, 95\% CI: 0.73-0.83) and for the score point system (C-statistic 0.74, 95\% CI: 0.69-0.80).

\section{DISCUSSION}

The present prospective and matched intervention trial offered a unique opportunity to examine whether pre-operative sociodemographic and health-related characteristics predict higher non-fatal self-harm and suicide rates following bariatric surgery. Our findings imply that male sex, psychiatric disorder history and sleep difficulties are relevant predictors in postbariatric patients. Although the risk of self-harm/suicide was still doubled in surgical patients compared to control patients after adjusting for these and other pre-treatment factors, we cannot exclude the possibility that the association is driven by selection bias, i.e. different patient characteristics in those who choose bariatric surgery instead of non-surgical obesity treatments.

Therefore, a challenging question that remains is to what extent higher rates of suicidal behaviors in post-bariatric patients reflect the effects of the performed surgery and to what

extent they reflect psychiatric and psychosocial vulnerabilities present prior to surgery. ${ }^{14}$ It is 
well-documented that psychiatric disorders are common in patients seeking and undergoing bariatric surgery ${ }^{24}$ and, in our study, history of self-harm and impaired HRQL at baseline were more frequent in surgical than control patients. Fully controlling for these differences is, however, difficult due to self-selection into surgery in our study and other studies in this area. Novel insights on this question might emerge from research investigating whether certain surgery-induced biological and behavioral changes (e.g. increased impulsivity due to heightened sensitivity to alcohol, hypoglycemia, reduced absorption of medications and nutrients, adverse quality of life or weight outcomes ${ }^{25,26}$ ) play a role in explaining the increased risk in post-bariatric patients.

The results from both multivariate models and interaction tests consistently suggested that particularly men can be at increased risk of self-harm/suicide after bariatric surgery. To our knowledge, this is the first controlled trial to demonstrate this. However, a few previous studies have reported a similar pattern of sex difference: a study based on Pennsylvania registers reported higher suicide rates in men (13.7 per 10000 person-years) than in women (5.2 per 10000 person-years) following bariatric surgery. ${ }^{13}$ Being male was also shown to predict a higher risk of self-harm/suicidal ideation in the 5 years after surgery. ${ }^{27}$ In the general population, a "gender paradox of suicidal behavior" has been reported: completed suicide is more prevalent in men, while non-fatal self-harm is more prevalent in women. ${ }^{28}$ Although we were not able to analyze these two outcomes separately (due to a low number of completed suicides: 7 in men and 6 in women), it is still possible that some of the mechanisms proposed to explain this gender paradox are relevant. For instance, under-diagnosed depression, substance-related problems, less than optimal treatment in psychiatric services and low treatment compliance may place particularly men at elevated risk of death by suicide. ${ }^{28}$ 
In line with the two previous studies in the bariatric population ${ }^{9,10}$ and a meta-analysis of 50 years of research in the general population, ${ }^{29}$ we found that three indicators of psychiatric disorder history (i.e. health-care visits for self-harm or mental disorders, psychiatric drug use) predicted higher self-harm/suicide rates after bariatric surgery. Moreover, forests plots indicated that the presence of self-harm or substance abuse history could further enhance the excess risk of these events in surgical patients compared to control patients. However, this observation should be interpreted with caution as the corresponding interactions were not statistically significant.

A novel and potentially important result from our research was that sleep difficulties emerged as an independent risk factor. The SOS study did not contain direct questions on sleep disturbances, but we were able to assess them indirectly by calculating a difference between self-reported sleep time per night and time spent in bed per night. In general, a growing body of literature suggests that sleep disturbances may confer increased risk for suicidal ideation, suicide attempts and death by suicide. ${ }^{30}$ This is highly plausible because sleep disturbances are closely intertwined with individual's psychosocial well-being: reduced sleep duration, for example, can be a marker of perceived stress, a symptom of depression, and a source of stress itself. ${ }^{31}$ Nonetheless, future studies using validated and more direct measures of sleep disturbances in bariatric patients should test whether our finding is replicable.

Compared to studies by Lagerros et al. ${ }^{9}$ and Morgan et al. ${ }^{10}$ that were solely based on registerbased information, we were able to examine a wider range of potential pre-operative risk factors. Estimates from the univariate models suggested that in addition to male sex, psychiatric disorder history and sleep difficulties various other health-related indicators were related to an elevated risk of self-harm/suicide in post-bariatric patients, including impaired 
self-rated HRQL, history of substance abuse, higher alcohol intake, daily smoking, lower BMI, and higher susceptibility to hunger. Moreover, together these risk factors from multiple domains had good predictive ability to identify those with adverse outcomes: a randomly selected patient with self-harm/suicide event could be distinguished from a randomly selected patient without such event with $80 \%$ accuracy. It is noteworthy that Gordon and colleagues ${ }^{27}$ recently identified a fairly similar set of pre-operative predictors (i.e. suicidality, being male, smoking, reporting greater pain, antidepressant use, psychiatric counseling, psychiatric hospitalizations) albeit in relation to post-operative self-harm/suicidal ideation, which is a less severe outcome.

Although prediction of suicidal behaviors at an individual level is challenging, ${ }^{29,32}$ we made an effort to create a scoring system for clinicians to identify those at highest risk of these events following bariatric surgery. All independent risk factors from the multivariate model, current depression or anxiety (as defined by the HADS total score $\geq 13^{33}$ ), and daily smoking (due to prior evidence ${ }^{27}$ ) were included in the score with the highest weight given to a history of self-harm or mental disorder (3 points for each). The results indicated that patients with 7 points had $10 \%$ probability of self-harm/suicide event in the 10 years after surgery, and the probability was over $20 \%$ in patients with score 9 or more. Thus, we suggest that a decision whether to operate should be made very carefully, and that it is essential that high-scoring patients who undergo surgery receive regular post-operative psychosocial monitoring to reduce the risk for future non-fatal and fatal self-harm. However, at the same time, it is crucial to ensure that the risk assessment and subsequent monitoring do not lead to adverse psychosocial consequences for high-risk patients (e.g. increased prejudice and stigma). This includes handling risk information confidentially. ${ }^{34}$ 
The lack of randomization and issues related to statistical power (particularly for interaction tests) are the main limitations of our study. The fact that surgery was self-selected makes it difficult to control for residual confounding, but this limitation is present in most bariatric surgery studies large enough to evaluate rare events. At the same time, our study better reflects the real clinical situation because it is typically the patient who asks for surgical treatment. In a randomized setting, it would not have been possible to identify self-harm behaviors associated with preference towards surgical obesity treatment. We may have missed a few non-fatal self-harm events before year 2001 when the NPR first started to cover both specialized out-patient and in-patient care. Other than this, the registers had excellent nationwide coverage. Because only a small number of suicides occurred during the follow-up, we decided to analyze the combined outcome of fatal and non-fatal self-harm events (the latter also representing rather severe events as defined in this study). We did not validate the scoring system externally and therefore its predictive power is likely to be over-estimated. The estimates in the control group were presented for comparison and should be interpreted with caution due to clearly lower event rate than in the surgery group. Notwithstanding these limitations, this is the first study with very long follow-up, to examine a wide-range of preoperative risk factors assessed using health examinations, questionnaires and national registers.

\section{Conclusions}

Our results indicate that pre-operative mental or psychiatric disorders are important risk factors for non-fatal self-harm and suicide in post-bariatric patients. Using information on psychiatric disorder history, sex, sleep difficulties, smoking, and current depression or anxiety, we created a simple-to-use prediction score to identify a high-risk group for these 
adverse events in a clinical setting. Future studies should test the predictive validity of this score in other bariatric patient samples. 


\section{REFERENCES}

1. Carlsson LM, Peltonen M, Ahlin S, et al. Bariatric surgery and prevention of type 2 diabetes in Swedish obese subjects. N Engl J Med 2012;367:695-704.

2. Ricci C, Gaeta M, Rausa E, et al. Long-term effects of bariatric surgery on type II diabetes, hypertension and hyperlipidemia: a meta-analysis and meta-regression study with 5-year follow-up. Obes Surg 2015;25:397-405.

3. Sjostrom L, Peltonen M, Jacobson P, et al. Bariatric surgery and long-term cardiovascular events. JAMA 2012;307:56-65.

4. Kwok CS, Pradhan A, Khan MA, et al. Bariatric surgery and its impact on cardiovascular disease and mortality: a systematic review and meta-analysis. Int J Cardiol 2014;173:20-28. 5. Adams TD, Gress RE, Smith SC, et al. Long-term mortality after gastric bypass surgery. N Engl J Med 2007;357:753-761.

6. Sjostrom L, Narbro K, Sjostrom CD, et al. Effects of bariatric surgery on mortality in Swedish obese subjects. N Engl J Med 2007;357:741-752.

7. Arterburn DE, Olsen MK, Smith VA, et al. Association between bariatric surgery and longterm survival. JAMA 2015;313:62-70.

8. Bhatti JA, Nathens AB, Thiruchelvam D, et al. Self-harm Emergencies After Bariatric Surgery: A Population-Based Cohort Study. JAMA Surg 2016;151:226-232.

9. Lagerros YT, Brandt L, Hedberg J, et al. Suicide, Self-harm, and Depression After Gastric Bypass Surgery: A Nationwide Cohort Study. Ann Surg 2017;265:235-243.

10. Morgan DJ, Ho KM. Incidence and Risk Factors for Deliberate Self-harm, Mental Illness, and Suicide Following Bariatric Surgery: A State-wide Population-based Linked-data Cohort Study. Ann Surg 2017;265:244-252. 
11. Neovius M, Bruze G, Jacobson P, et al. Risk of suicide and non-fatal self-harm after bariatric surgery: results from two matched cohort studies. Lancet Diabetes Endocrinol 2018;6:197-207.

12. Castaneda D, Popov VB, Wander P, et al. Risk of Suicide and Self-harm Is Increased After Bariatric Surgery-a Systematic Review and Meta-analysis. Obes Surg 2019;29:322-333.

13. Tindle HA, Omalu B, Courcoulas A, et al. Risk of suicide after long-term follow-up from bariatric surgery. Am J Med 2010;123:1036-1042.

14. Courcoulas A. Who, Why, and How? Suicide and Harmful Behaviors After Bariatric Surgery. Ann Surg 2017;265:253-254.

15. Pocock SJ, Simon R. Sequential treatment assignment with balancing for prognostic factors in the controlled clinical trial. Biometrics 1975;31:103-115.

16. Keating C, Neovius M, Sjoholm K, et al. Health-care costs over 15 years after bariatric surgery for patients with different baseline glucose status: results from the Swedish Obese Subjects study. Lancet Diabetes Endocrinol 2015;3:855-865.

17. Lindroos AK, Lissner L, Sjostrom L. Validity and reproducibility of a self-administered dietary questionnaire in obese and non-obese subjects. Eur J Clin Nutr 1993;47:461-481.

18. Stunkard AJ, Messick S. The three-factor eating questionnaire to measure dietary restraint, disinhibition and hunger. J Psychosom Res 1985;29:71-83.

19. Davies AR, Sherbourne CR, Peterson JR, et al. Scoring Manual: Adult Health Status and Patient Satisfaction Measures Used in RAND's Health Insurance Experiment. Santa Monica, CA: RAND Corporation; 1988.

20. Sjoberg L, Svensson E, Persson LO. The measurement of mood. Scand J Psychol 1979;20:1-18.

21. Zigmond AS, Snaith RP. The hospital anxiety and depression scale. Acta Psychiatr Scand 1983;67:361-370. 
22. Bergner M, Bobitt RA, Carter WB, et al. The sickness impact profile: development and final revision of a health status measure. Med Care 1981;19:787-805.

23. Karlsson J, Taft C, Sjostrom L, et al. Psychosocial functioning in the obese before and after weight reduction: construct validity and responsiveness of the Obesity-related Problems scale. Int J Obes Relat Metab Disord 2003;27:617-630.

24. Dawes AJ, Maggard-Gibbons M, Maher AR, et al. Mental Health Conditions Among Patients Seeking and Undergoing Bariatric Surgery: A Meta-analysis. JAMA 2016;315:150163.

25. Mitchell JE, Crosby R, de Zwaan M, et al. Possible risk factors for increased suicide following bariatric surgery. Obesity (Silver Spring) 2013;21:665-672.

26. Spittal MJ, Fruhbeck G. Bariatric surgery: many benefits, but emerging risks. Lancet Diabetes Endocrinol 2018;6:161-163.

27. Gordon KH, King WC, White GE, et al. A longitudinal examination of suicide-related thoughts and behaviors among bariatric surgery patients. Surg Obes Relat Dis 2019;15:269278.

28. Schrijvers DL, Bollen J, Sabbe BG. The gender paradox in suicidal behavior and its impact on the suicidal process. J Affect Disord 2012;138:19-26.

29. Franklin JC, Ribeiro JD, Fox KR, et al. Risk factors for suicidal thoughts and behaviors: A meta-analysis of 50 years of research. Psychol Bull 2017;143:187-232.

30. Bernert RA, Kim JS, Iwata NG, et al. Sleep disturbances as an evidence-based suicide risk factor. Curr Psychiatry Rep 2015;17:554-015-0554-4.

31. Geiker NRW, Astrup A, Hjorth MF, et al. Does stress influence sleep patterns, food intake, weight gain, abdominal obesity and weight loss interventions and vice versa? Obes Rev 2018;19:81-97. 
32. Belsher BE, Smolenski DJ, Pruitt LD, et al. Prediction Models for Suicide Attempts and Deaths: A Systematic Review and Simulation. JAMA Psychiatry 2019;76:642-651.

33. Hinz A, Brahler E. Normative values for the hospital anxiety and depression scale (HADS) in the general German population. J Psychosom Res 2011;71:74-78.

34. Passos IC, Ballester P. Positive Predictive Values and Potential Success of Suicide Prediction Models. JAMA Psychiatry 2019. 


\section{FIGURE LEGENDS}

Figure 1. Cumulative incidence function from competing risk regression model for nonfatal self-harm and suicide in the Swedish Obese Subjects study: total sample and by sex. Test of sex $\times$ treatment group interaction: $\mathrm{p}=0.0495$ unadjusted and $\mathrm{p}=0.007$ adjusted for age, study inclusion year, history of self-harm, history of mental disorder, psychiatric drug use, and sleep difficulties. Abbreviations: subHR, sub-hazard ratio; CI, confidence interval. 
Table 1. Baseline descriptive characteristics by the treatment group in the Swedish Obese Subjects study

\begin{tabular}{|c|c|c|c|c|c|}
\hline \multirow[b]{2}{*}{ Variable } & \multicolumn{2}{|c|}{ Control $(\mathrm{N}=2040)$} & \multicolumn{2}{|c|}{ Surgery $(N=2007)^{a}$} & \multirow[b]{2}{*}{$\mathrm{p}$ value $\mathrm{b}^{\mathrm{b}}$} \\
\hline & Mean / \% & $\mathrm{SD} / \mathrm{N}$ & Mean / \% & $\mathrm{SD} / \mathrm{N}$ & \\
\hline \multicolumn{6}{|l|}{ Sociodemographics } \\
\hline Age (years) & 48.7 & 6.3 & 47.2 & 5.9 & $<0.001$ \\
\hline Men $(\%)$ & 29.1 & 593 & 29.2 & 587 & 0.917 \\
\hline Married or partner $(\%)$ & 75.3 & 1527 & 72.9 & 1460 & 0.098 \\
\hline Education & & & & & $<0.001$ \\
\hline $\operatorname{Basic}(\%)$ & 59.9 & 1222 & 69.4 & 1393 & \\
\hline Upper secondary (\%) & 19.0 & 387 & 17.8 & 357 & \\
\hline University (\%) & 21.1 & 431 & 12.8 & 257 & \\
\hline \multicolumn{6}{|l|}{ Health status } \\
\hline Diabetes (\%) & 12.9 & 263 & 17.2 & 344 & $<0.001$ \\
\hline $\operatorname{BMI}\left(\mathrm{kg} / \mathrm{m}^{2}\right)$ & 40.1 & 4.7 & 42.4 & 4.5 & $<0.001$ \\
\hline Joint pain $(\%)$ & 55.4 & 1130 & 67.9 & 1362 & $<0.001$ \\
\hline \multicolumn{6}{|l|}{ History of psychiatric disorder } \\
\hline Self-harm $(\%)$ & 1.9 & 38 & 3.4 & 69 & 0.002 \\
\hline Substance abuse (\%) & 2.5 & 50 & 2.9 & 58 & 0.435 \\
\hline Mental disorder $(\%)$ & 8.6 & 176 & 9.9 & 198 & 0.175 \\
\hline Psychiatric drug use (\%) & 10.8 & 220 & 12.2 & 244 & 0.183 \\
\hline \multicolumn{6}{|l|}{ Lifestyle behaviors } \\
\hline Daily smokers (\%) & 20.8 & 422 & 25.8 & 518 & $<0.001$ \\
\hline Alcohol intake (g/day) & 5.3 & 8.1 & 5.2 & 7.2 & 0.632 \\
\hline Sleep difficulties & 1.0 & 1.0 & 1.0 & 1.0 & 0.114 \\
\hline \multicolumn{6}{|l|}{ (awake in bed/night, h) } \\
\hline Cognitive restraint $(\text { TFEQ })^{\mathrm{c}}$ & 9.8 & 4.7 & 8.6 & 4.3 & $<0.001$ \\
\hline Disinhibition $(\mathrm{TFEQ})^{\mathrm{c}}$ & 8.7 & 3.7 & 9.5 & 3.6 & $<0.001$ \\
\hline Hunger $(\text { TFEQ })^{\mathrm{c}}$ & 6.5 & 3.5 & 7.3 & 3.5 & $<0.001$ \\
\hline \multicolumn{6}{|l|}{ Self-rated HRQL ${ }^{d}$} \\
\hline Perceived health & 57.5 & 25.7 & 50.1 & 24.7 & $<0.001$ \\
\hline Anxiety & 5.4 & 4.6 & 6.0 & 4.5 & $<0.001$ \\
\hline Depression & 4.1 & 3.5 & 5.3 & 3.6 & $<0.001$ \\
\hline Overall mood & 3.0 & 0.6 & 2.9 & 0.6 & $<0.001$ \\
\hline Obesity-related problems & 41.4 & 26.9 & 58.9 & 26.7 & $<0.001$ \\
\hline Social interaction & 9.1 & 12.0 & 14.0 & 13.6 & $<0.001$ \\
\hline
\end{tabular}

Abbreviations: HRQL, health-related quality of life; TFEQ, Three-Factor Eating Questionnaire.

${ }^{\text {a }}$ Type of surgery: nonadjustable or adjustable banding (376 patients), vertical banded gastroplasty (1365 patients), and gastric bypass (266 patients).

${ }^{b}$ Differences between the treatment groups were tested using t-tests (continuous variables) or Fisher's exact tests (categorical variables). 
${ }^{c}$ Higher scores represent more cognitive restraint (score range 0-21), disinhibition (score range 0-16), and hunger (score range $0-14$ ).

${ }^{\mathrm{d}}$ Perceived health: higher scores represent better perceived health (score range 0-100). Social interaction and obesityrelated problems: higher scores represent more dysfunction (score range 0-100). Overall mood: higher scores represent more positive mood states (score range 1-4). Anxiety and depression: higher scores represent more symptoms (score range 0-21). 
Table 2. Results from multivariate competing risk regression models predicting the risk of non-fatal self-harm and suicide in the Swedish Obese Subjects study ${ }^{\mathrm{a}}$

\begin{tabular}{|c|c|c|c|c|c|c|c|}
\hline \multirow[b]{2}{*}{ Risk factor } & & \multicolumn{2}{|c|}{$\begin{array}{l}\text { Surgery + Control } \\
(\mathrm{N}=4047)\end{array}$} & \multicolumn{2}{|c|}{$\begin{array}{l}\text { Control } \\
(\mathrm{N}=2040)\end{array}$} & \multicolumn{2}{|c|}{$\begin{array}{l}\text { Surgery } \\
(\mathrm{N}=2007)\end{array}$} \\
\hline & & subHR & {$[95 \% \mathrm{CI}]$} & subHR & {$[95 \% \mathrm{CI}]$} & subHR & {$[95 \% \mathrm{CI}]$} \\
\hline \multirow[t]{2}{*}{ Treatment group } & Control & 1.00 & & & & & \\
\hline & Surgery & $1.98 * * *$ & [1.34-2.93] & & & & \\
\hline Study inclusion year & & $1.07 * *$ & [1.02-1.12] & 1.01 & [0.93-1.10] & $1.09 * *$ & [1.03-1.16] \\
\hline \multirow[t]{2}{*}{ Sex } & Women & 1.00 & & 1.00 & & 1.00 & \\
\hline & Men & 1.24 & [0.82-1.88] & 0.60 & {$[0.25-1.44]$} & $1.75^{*}$ & [1.07-2.85] \\
\hline BMI $\left(\mathrm{kg} / \mathrm{m}^{2}\right)$ & & 0.98 & {$[0.95-1.01]$} & 0.97 & [0.91-1.03] & 0.98 & [0.94-1.02] \\
\hline \multirow[t]{2}{*}{ Joint pain } & No & 1.00 & & 1.00 & & 1.00 & \\
\hline & Yes & 1.18 & {$[0.77-1.79]$} & 1.76 & {$[0.86-3.60]$} & 0.93 & {$[0.56-1.54]$} \\
\hline \multirow[t]{2}{*}{ History of self-harm } & No & 1.00 & & 1.00 & & 1.00 & \\
\hline & Yes & $2.17 * *$ & {$[1.22-3.85]$} & 1.14 & {$[0.26-5.02]$} & $2.84 * *$ & {$[1.48-5.45]$} \\
\hline \multirow[t]{2}{*}{ History of substance abuse } & No & 1.00 & & 1.00 & & 1.00 & \\
\hline & Yes & 0.90 & {$[0.47-1.73]$} & 0.50 & {$[0.12-2.06]$} & 1.08 & [0.49-2.36] \\
\hline \multirow[t]{2}{*}{ History of mental disorder } & No & 1.00 & & 1.00 & & 1.00 & \\
\hline & Yes & $3.11 * * *$ & {$[1.95-4.96]$} & $4.00 * * *$ & {$[1.80-8.87]$} & $3.11 * * *$ & {$[1.79-5.41]$} \\
\hline \multirow[t]{2}{*}{ Psychiatric drug use } & No & 1.00 & & 1.00 & & 1.00 & \\
\hline & Yes & $1.77 * *$ & {$[1.19-2.64]$} & 1.68 & {$[0.80-3.55]$} & $2.02 * *$ & {$[1.25-3.26]$} \\
\hline \multirow[t]{2}{*}{ Smoking } & No & 1.00 & & 1.00 & & 1.00 & \\
\hline & Yes & 1.36 & [0.93-1.99] & 1.38 & {$[0.72-2.63]$} & 1.35 & {$[0.83-2.20]$} \\
\hline Alcohol intake (g/day) & & 1.02 & {$[1.00-1.04]$} & 1.02 & {$[0.99-1.06]$} & 1.02 & {$[0.99-1.05]$} \\
\hline $\begin{array}{l}\text { Sleep difficulties } \\
\text { (awake in bed/night, h) }\end{array}$ & & $1.35 * * *$ & {$[1.18-1.55]$} & $1.52 * * *$ & {$[1.25-1.85]$} & $1.27 * *$ & {$[1.06-1.52]$} \\
\hline Hunger (TFEQ) $)^{b}$ & & 1.02 & {$[0.97-1.07]$} & 0.99 & {$[0.91-1.07]$} & 1.03 & {$[0.96-1.10]$} \\
\hline Perceived health ${ }^{\mathrm{b}}$ & & 1.00 & {$[0.99-1.01]$} & 0.99 & {$[0.97-1.00]$} & 1.00 & {$[0.99-1.01]$} \\
\hline Anxiety $^{\mathrm{b}}$ & & 1.04 & [0.99-1.09] & 0.98 & [0.90-1.07] & 1.06 & {$[0.99-1.13]$} \\
\hline Depression $^{\mathrm{b}}$ & & 0.96 & {$[0.90-1.04]$} & 0.92 & [0.81-1.04] & 0.98 & {$[0.90-1.07]$} \\
\hline Overall mood ${ }^{\mathrm{b}}$ & & 0.90 & {$[0.52-1.55]$} & 0.80 & {$[0.33-1.97]$} & 0.90 & {$[0.43-1.88]$} \\
\hline Obesity-related problems ${ }^{\mathrm{b}}$ & & 1.00 & {$[0.99-1.00]$} & 1.00 & {$[0.98-1.01]$} & 1.00 & {$[0.99-1.01]$} \\
\hline Social interaction $^{\mathrm{b}}$ & & 1.00 & {$[0.99-1.02]$} & 1.01 & [0.99-1.04] & 1.00 & [0.98-1.01] \\
\hline
\end{tabular}

$* * * \mathrm{P}<0.001, * * \mathrm{P}<0.01, * \mathrm{P}<0.05$.

Abbreviations: subHR, sub-hazard ratio; CI, confidence interval; TFEQ, Three-Factor Eating Questionnaire.

aDiscrimination of the models: Harrel's C $=0.80$ (95\% CI: 0.76-0.83) for surgery + control, Harrel's C $=0.79$ (95\% CI:

0.72-0.86) for control, and Harrel's C=0.80 (95\% CI: 0.75-0.84) for surgery.

${ }^{\mathrm{b}}$ Hunger (TFEQ): higher scores represent more hunger (score range 0-14). Perceived health: higher scores represent better perceived health (score range 0-100). Social interaction and obesity-related problems: higher scores represent 
more dysfunction (score range 0-100). Overall mood: higher scores represent more positive mood states (score range 14). Anxiety and depression: higher scores represent more symptoms (score range 0-21). 
Table 3. Multivariate competing risk regression model for non-fatal self-harm and suicide events in the SOS surgery group, resulting score points for each risk factor, and total score points with estimated probabilities for an event over 10 years $^{\mathrm{a}}$

\begin{tabular}{|c|c|c|c|c|c|c|c|}
\hline \multirow[b]{2}{*}{ Risk factor } & \multicolumn{5}{|c|}{ Estimates from regression model and resulting score points } & \multicolumn{2}{|c|}{ Total score points and corresponding probability } \\
\hline & Category & subHR & {$[95 \% \mathrm{CI}]$} & $\mathrm{p}$ value & Points $^{\mathrm{d}}$ & Total points ${ }^{\mathrm{e}}$ & 10-year probability \\
\hline \multirow[t]{2}{*}{ Sex } & Women & 1.00 & & & 0 & 0 & 0.9 \\
\hline & Men & 2.12 & [1.40-3.19] & $<0.001$ & 2 & 1 & 1.1 \\
\hline \multirow[t]{2}{*}{ Smoking } & No & 1.00 & & & 0 & 2 & 1.6 \\
\hline & Yes & 1.43 & {$[0.91-2.25]$} & 0.125 & 1 & 3 & 2.3 \\
\hline History of self-harm & No & 1.00 & & & 0 & 4 & 2.9 \\
\hline \multirow[t]{2}{*}{ History of mental disorder } & No & 1.00 & & & 0 & 6 & 7.5 \\
\hline & Yes & 2.97 & [1.88-4.69] & $<0.001$ & 3 & 7 & 10.2 \\
\hline \multirow[t]{2}{*}{ Psychiatric drug use } & No & 1.00 & & & 0 & 8 & 13.0 \\
\hline & Yes & 2.33 & {$[1.50-3.63]$} & $<0.001$ & 2 & 9 or more & $>20$ \\
\hline Sleep difficulties ${ }^{\mathrm{f}}$ & No & 1.00 & & & 0 & & \\
\hline
\end{tabular}

Abbreviations: SOS, Swedish Obese Subjects; subHR, sub-hazard ratio; CI, confidence interval.

${ }^{a}$ Total of 2007 patients who underwent bariatric surgery with 98 non-fatal self-harm or suicide events.

bBaseline cumulative incidence function from competing risk regression model at 10 year: 0.015589 . Discrimination of the original model: Harrel's C=0.78 (95\% CI: $0.73-$

$0.83)$ in 5000 bootstrap samples.

'Discrimination of the score: Harrel's C=0.74 (95\% CI: 0.69-0.80) in 5000 bootstrap samples.

${ }^{\mathrm{d}}$ One score point corresponds to a half of the risk difference between men and women.

${ }^{\mathrm{e}}$ Total score points can vary between 0 and 13 .

${ }_{\mathrm{f}}^{\mathrm{f}}$ Awake 1 hour or more in bed per night.

${ }^{\text {g}}$ The Hospital Anxiety and Depression Scale total score $\geq 13$ as proposed by Hinz and Brähler. ${ }^{33}$ 
Total

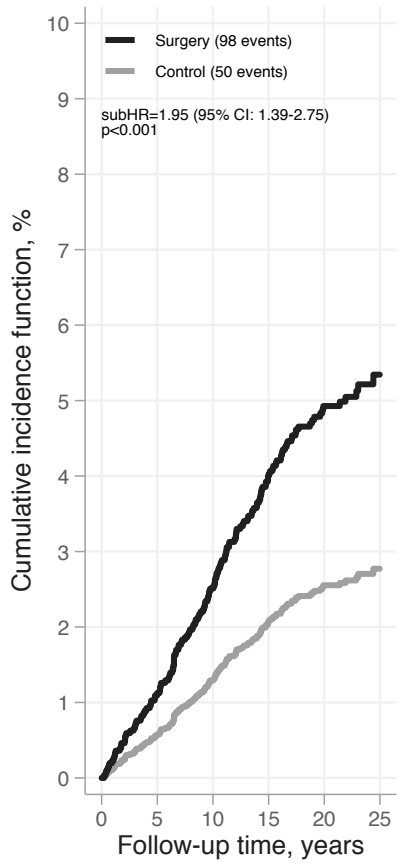

\section{Women}

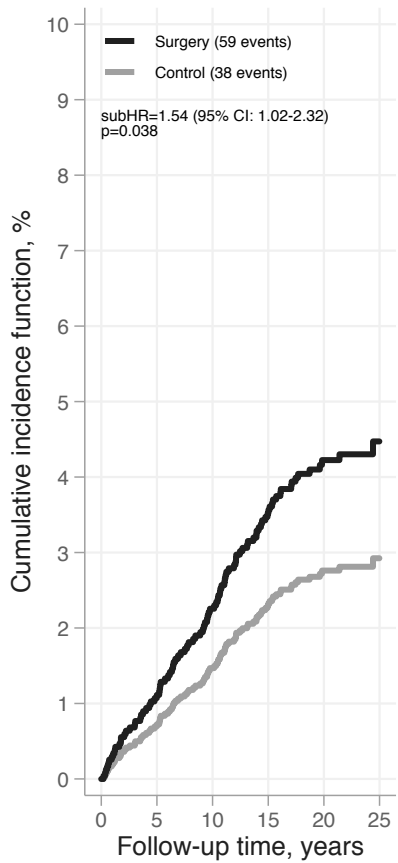

Men

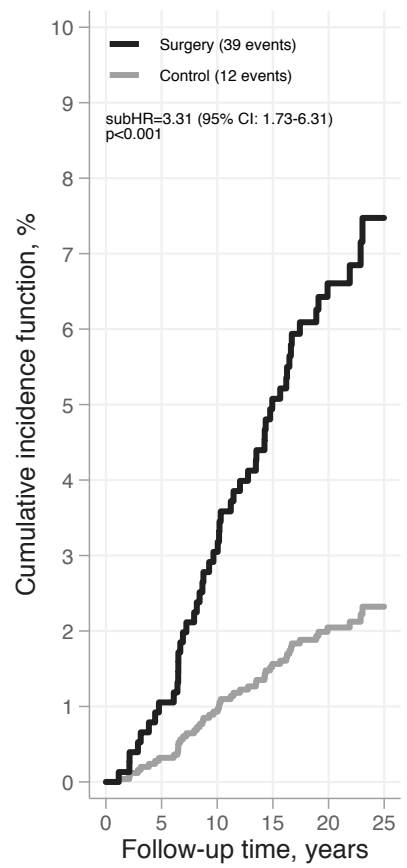

